\title{
Constructive universal central limit theorems based on interacting Fock spaces
}

\begin{abstract}
Cabanal-Duvillard and Ionescu [1] have proved that any symmetric probability measure with moments of any order can be obtained as central limit theorem of self-adjoint, weakly independent and symmetrically distributed (in a quantum sense) random variables. Results of this type will be called "universal central limit theorem".

Using Interacting Fock Space (IFS) techniques we extend this result in two directions: (i) we prove that the random variables can be taken to be generalized Gaussian in the sense of Accardi and Bożejko [3] and we give a realization of such random variables as sums of creation, annihilation and preservation operators acting on an appropriate IFS; (ii) we extend the above mentioned result to the non symmetric case. The non trivial difference between the symmetric and the non symmetric case is explained at the end of the introduction below.
\end{abstract}

\section{Accardi}

Centro V. Volterra

Università Roma Tor Vergata

00133 Rome, Italy

accardi@volterra.mat.uniroma2.it
V. Crismale

Dip. di Matematica

Università di Bari

70125 Bari, Italy

crismalev@dm.uniba.it
Y.G. Lu

Dip. di Matematica

Università di Bari

70125 Bari, Italy

lu@dm.uniba.it

Keywords: central limit theorem, quantum probability, interacting Fock spaces.

AMS Subject Classification: 60B99, 46L53 


\section{$\S 1$ Introduction}

The main technical tool, used in the present paper, is the Interacting Fock Space (IFS). This notion emerged from the stochastic limit of quantum electrodynamics (QED) without dipole approximation [1].

The functoriality of the construction of the IFS suggested, since the beginning of the theory, that it might be a natural tool to construct examples of inequivalent notions of stochastic independence.

On the other hand, the proliferation of notions of stochastic independence has motivated the development of different points of view concerning these notions. In particular we mention:

(i) the axiomatic point of view, based on various notions of coproduct and developed by Schürmann [23], Speicher [26], Ben Ghorbal [6], Muraki [22],

(ii) the reductionistic point of view, developed by Lenczewski, which reduces all notions of independence to tensor independence [17], see also [13],

(iii) the individualistic point of view, which concentrates on a specific notion of independence and extensively develops the corresponding probability theory in analogy with the classical one. This has been followed by Voiculescu [27], Bożejko and Speicher [10], Speicher [24], Bercovici [8], for free probability; by Lu [18, 19] and especially Muraki [21], for monotone probability; by Speicher and Woroudi [25], Ben Ghorbal and Schürmann [7] for boolean probability;

(iv) the constructive approach, which emphasizes concrete and explicit representations of the random variables involved. Examples of this approach can be found in the paper [9] by Bozejko, Kümmerer, Speicher on $q$-Gaussian processes and in several other papers of the Polish QPschool; in the papers by Accardi, Hashimoto, Obata [4, Hashimoto [15], Hashimoto, Hora and Obata [16], Lu [19], which use the IFS as a basic tool.

From the Lenczewski approach [17] it begun to emerge the idea that the various notions of quantum independence are in fact masked forms of classical dependencies. This point of view received two independent confirmations: 
one in the result, proved by Cabanal Duvillard and Ionescu [11, that any symmetric probability measure on $\mathbb{R}$, with moments of any order can be obtained as the central limit distribution, in the sense of convergence in moments of a sequence of symmetrically distributed algebraic random variables satisfying a notion of stochastic independence. Another one was obtained by Accardi and Bożejko 3] who introduced a universal convolution and proved that every symmetric probability law on $\mathbb{R}$ with moments of any order is infinitely divisible with respect to this convolution.

The result of [3] was based on interacting Fock space techniques while the result of Cabanal-Duvillard and Ionescu exploited the combinatorial techniques of [12] combined with an extension of the notion of $(\varphi, \psi)$-independence, due to Bożejko and Speicher [10]. This construction was generalized by Mlotkowski [20]. Between the results obtained in [3, 11, 20] and the central limit theorem proved in the present paper, there are three main differences:

1. we prove that any mean-zero distribution with moments of all orders, not only the symmetric ones, can be obtained as central limits, in the sense of convergence of moments, of self-adjoint random variables;

2. we explicitly realize both the approximating random variables and their limits as sums of creation, annihilation and number operators in suitable IFS;

3. with respect to the reference state, the random variables considered here do not satisfy the weak independence condition, used in [11] neither, in the case of non symmetric distribution, the symmetrically distributed condition, used in the same paper.

The present paper also suggests a general notion of independence, naturally abstracting the properties of the special class of interacting Fock spaces which is used here (cf. section 4), and different from the notion of weak independence in the sense of Cabanal-Duvillard and Ionescu. This development will be discussed in [5].

It was known from the Accardi-Bożejko paper [3] that the mixed moments of any probability measure with moments of any order can be expressed in terms of singletons and (non crossing) pair partitions (Gaussianization) and that the symmetric case is characterized by the absence of singletons. The functoriality of the IFS construction provides a natural and easy way to extend this construction from single random variables to processes. 
A nontrivial difference between the symmetric and the non symmetric central limit theorem is that we are unable to prove the latter with identically distributed random variables. We were able to reduce this dishomogeneity to a simple multiplication by a constant (cf. section (\$5) formula (5.2)), but not to eliminate it completely. It is not known whether this is a limitation of our method or the manifestation of an intrinsic difference between the two cases. The analogy with the classical case suggests the conjecture that the latter hypothesis is true.

This paper is organized as follows in section $\$ 2$ we recall the definition of IFS and of the basic operators acting on a such spaces, i.e. creation, annihilation and preservation operators. In section $\$ 3$ one give a formula for the moments of a quantum stochastic process of the form of $A(f)+$ $A^{+}(f)+\Lambda_{\alpha}$. In section $\S 4$, we introduce the notion of "1-mode-type IFS" (1-MT-IFS) and show that on any 1 -MT-IFS, the vacuum distribution of $A(f)+A^{+}(f)+\Lambda_{\alpha}$ depends only on the module of $f$ but not on $f$ itself. This is the main difference between IFS and 1-MT-IFS, for more details see [19]. Section $\$ 5$ is devoted to the proof of our main result i.e. Theorem 5.1. First, we prove an estimate on the mixed moments (Lemma 5.2) which allows to apply Lemma 2.4 of [4]. This, combined with a quantum extension of the moment formula proved in Theorem 5.1 of [3], allows to conclude the proof of our Theorem 5.1 in the symmetric case. Up to this point we work with identically distributed random variables. Then we extend the proof to the non symmetric case trying to emphasize the steps of the proof that prevent us from using only identically distributed random variables (cf. formula (5.12)). Corollary (5.1) specializes the above result to the associated classical process and corresponds to the nonsymmetric extension of the Cabanal-Duvillard and Ionescu result.

\section{$\S 2$ Standard IFS}

In this and following sections we recall from [2] the definition and some properties of the standard IFS. Then, using these notions, we formulate the main results of this paper.

Definition 2.1 Let $(X, \mathcal{X}, \mu)$ be a measure space and let $\left\{\lambda_{n}\right\}_{n=1}^{\infty}$ be a family of functions with the following properties:

(i) for any $n \in \mathbb{N}, \lambda_{n}:\left(X^{n}, \mathcal{X}^{n}\right) \rightarrow \mathbb{R}_{+}$is bounded, positive, measurable; 
(ii) for any measurable function $F_{n}:\left(X^{n}, \mathcal{X}^{n}\right) \rightarrow \mathbb{C}$ if

$$
\int\left|F_{n}\left(x_{n}, \ldots, x_{1}\right)\right|^{2} \lambda_{n}\left(x_{n}, \ldots, x_{1}\right) \mu\left(d x_{n}\right) \cdots \mu\left(d x_{1}\right)=0
$$

then for any measurable function $f:(X, \mathcal{X}) \rightarrow \mathbb{C}$,

$$
\int|f(x)|^{2}\left|F_{n}\left(x_{n}, \ldots, x_{1}\right)\right|^{2} \lambda_{n+1}\left(x, x_{n}, \ldots, x_{1}\right) \mu(d x) \mu\left(d x_{n}\right) \cdots \mu\left(d x_{1}\right)=0
$$

We define, for each $n \in \mathbb{N}$, the (not necessarily finite) measure $\mu_{n}$ on $X^{n}$ by

$$
\mu_{n}(E):=\int_{E} \lambda_{n}\left(x_{n}, \ldots, x_{1}\right) \mu\left(d x_{n}\right) \cdots \mu\left(d x_{1}\right), \quad \text { for } E \in X^{n},
$$

and the associated $L^{2}-$ space:

$$
H_{n}:=\mathbf{L}^{2}\left(\mathbb{R}^{n}, \mu_{n}\right), \quad \forall n \geq 2
$$

with pre-scalar product such that for any $F_{n}, G_{n} \in H_{n}$

$$
\left\langle F_{n}, G_{n}\right\rangle:=\int \lambda_{n}\left(x_{n}, \ldots, x_{1}\right)\left(\overline{F_{n}} G_{n}\right)\left(x_{n}, \ldots, x_{1}\right) \mu\left(d x_{n}\right) \cdots \mu\left(d x_{1}\right) .
$$

By taking the quotient and completing $H_{n}$ becomes an Hilbert space and with the convention that

$$
H_{0}:=\mathbb{C}, \quad H:=H_{1}
$$

The space

$$
\Gamma\left(H,\left\{\lambda_{n}\right\}_{n}\right):=\bigoplus_{n=0}^{\infty} H_{n}, \quad \Phi:=1 \oplus 0 \oplus 0 \oplus \cdots
$$

is called the (standard) interacting Fock space with weight functions $\left\{\lambda_{n}\right\}_{n=1}^{\infty}$. In particular if the $\left\{\lambda_{n}\right\}_{n=1}^{\infty}$ are constant, then the corresponding space $\Gamma\left(H,\left\{\lambda_{n}\right\}_{n}\right)$ is called a 1-mode type free interacting Fock space (1MT-IFS in short).

Definition 2.2 On the standard interacting Fock space $\Gamma\left(H,\left\{\lambda_{n}\right\}_{n}\right)$, for any $f \in H$, for any $n \in \mathbb{N}$ and for any $F_{n} \in H_{n}$ the creation operator $A^{+}(f)$ is defined by:

$$
\left(A^{+}(f) F_{n}\right)\left(x_{n+1}, x_{n}, \ldots, x_{1}\right):=f\left(x_{n+1}\right) \cdot F_{n}\left(x_{n}, \ldots, x_{1}\right)
$$


It can be proved that $A^{+}(f)$ is well defined as a linear operator $A_{f}^{+}: H_{n} \rightarrow$ $H_{n+1}$ and that it has an adjoint $A(f): H_{n} \rightarrow H_{n-1}$ (on an appropriate domain, $c f .[2])$ called the annihilation operator

$$
A(f):=\left(A^{+}(f)\right)^{*}
$$

If we assume that for any natural integer $n$ and $k=1,2, \ldots, n+1$, there is a $C_{n, k}$ such that

$$
\lambda_{n+1}\left(x_{n+1}, \ldots, x_{2}, x_{1}\right) \leq C_{n, k} \lambda_{1}\left(x_{k}\right) \cdot \lambda_{n}\left(x_{n+1}, \ldots, x_{k+1}, x_{k-1}, \ldots, x_{1}\right)
$$

then, for each $n \in \mathbb{N}, A^{+}(f): H_{n} \rightarrow H_{n+1}$ is a linear bounded operator (see [2] for more details).

Definition 2.3 For any $X \in \mathbf{B}(H)$, for any $\alpha=\left\{\alpha_{n}\right\}_{n=0}^{\infty} \subset \mathbb{R}$ with $\alpha_{0}:=0$, for any $n \in \mathbb{N}$ and for any $f_{1}, \ldots, f_{n} \in H$, one defines

$$
\Lambda_{\alpha}(X)\left(f_{1} \otimes f_{2} \otimes \cdots \otimes f_{n}\right):=\alpha_{n}\left(X f_{1}\right) \otimes f_{2} \otimes \cdots \otimes f_{n}
$$

the number operator with intensity $\left(\left\{\alpha_{n}\right\}_{n=0}^{\infty}, X\right)$.

For simplicity we shall adopt throughout this paper the following conventions:

- if $X$ is the identity operator, $\Lambda_{\alpha}(X)$ will be denoted simply

$$
\Lambda_{\alpha}:=\Lambda_{\alpha}(1)
$$

- for any $f \in H$ we write

$$
\Lambda_{\alpha}(f):=\Lambda_{\alpha}\left(M_{f}\right)
$$

where $M_{f}$ is the multiplication by $f$, i.e. $M_{f} g:=f g$, for any $g \in H$.

For any $\varepsilon \in\{-1,0,1\}, f \in H, \alpha \in \ell^{\infty}\left(\mathbb{R}^{\mathbb{N}}\right)$ we will denote

$$
A^{\varepsilon}(f)=A_{f}^{\varepsilon}:=\left\{\begin{array}{l}
A^{+}(f), \text { if } \varepsilon=1 \\
\Lambda_{\alpha}(f), \text { if } \varepsilon=0 \\
A(f), \text { if } \varepsilon=-1
\end{array}\right.
$$

The properties of standard IFS imply that, for any $n \in \mathbb{N}, \varepsilon(1), \ldots, \varepsilon(n) \in$ $\{-1,1\}, f_{1}, \ldots, f_{n} \in H$, for any sequence $\left(\alpha_{n}\right)$ with $\alpha_{0}=0$, and for any $X \in \mathbf{B}(H)$, the following identity holds (in the sense that both sides are well defined and the identity holds):

$$
\Lambda_{\alpha}(X) A^{\varepsilon(n)}\left(f_{n}\right) \cdots A^{\varepsilon(1)}\left(f_{1}\right) \Phi=\alpha_{m} A^{\varepsilon(n)}\left(g_{n}\right) \cdots A^{\varepsilon(1)}\left(g_{1}\right) \Phi
$$

where 
- among the vectors $g_{1}, \ldots, g_{n}$, there is exactly one index $i$ such that

$$
g_{i}=X f_{i}, \quad g_{j}=f_{j} \quad \forall j \neq i
$$

- also the index $m$ is uniquely determined by the family $\{\varepsilon(1), \ldots, \varepsilon(n)\}$.

As a consequence any product of creation, annihilation and number operators applied to the vacuum can be always reduced to a multiple of a product of only creation and annihilation operators applied to the vacuum.

The $\Phi$-statistics of the operator stochastic process $\left\{A(f), A^{+}(g), \Lambda_{\alpha}(X)\right.$ : $f, g \in H, X \in \mathbf{B}(H)\}$ is coded into its mixed moments

$$
\left\langle A^{\varepsilon(1)}\left(f_{1}\right) \cdots A^{\varepsilon(n)}\left(f_{n}\right)\right\rangle:=\left\langle\Phi, A^{\varepsilon(1)}\left(f_{1}\right) \cdots A^{\varepsilon(n)}\left(f_{n}\right) \Phi\right\rangle
$$

through the algebraic rules (2.5) and:

$$
A(f) \Phi=0, \quad\left\langle A(f) A^{+}(g)\right\rangle=\langle f, g\rangle
$$

The extension of the Cabanal-Duvillard-Ionescu theorem, mentioned in the introduction, can be formulated as follows (cf. Corollary (5.1)): for any mean-zero probability measure $\mu$ on $\mathbb{R}$ with moments of any order, there exists an IFS $\Gamma\left(H,\left\{\lambda_{n}\right\}_{n}\right)$ and a family of operator random variables $\left\{Q_{k}\right\}_{k=1}^{\infty}$ such that, for any $m \in \mathbb{N}$

$$
\lim _{N \rightarrow \infty}\left\langle\left(\frac{1}{\sqrt{N}} \sum_{k=1}^{N} Q_{k}\right)^{m}\right\rangle=\int x^{m} d \mu
$$

Moreover, both the family $\left\{\lambda_{n}\right\}_{n}$ and the construction of $\left\{Q_{k}\right\}_{k=1}^{\infty}$ are determined by the Jacobi parameters of the measure $\mu$. This result will be obtained as a corollary of a more general quantum central limit theorem (cf. Theorem (5.1)).

\section{$\S 3$ Moments of creators, annihilators and num- ber operators in IFS}

Lemma 3.1 For any $n \in \mathbb{N}$ and $\varepsilon$ belonging to the set

$$
\{-1,0,1\}^{n}:=\{\varepsilon=(\varepsilon(n), \cdots, \varepsilon(1)): \varepsilon(i) \in\{-1,0,1\}, \forall i=1, \ldots, n\}
$$


i) if among $\left\{A^{\varepsilon(n)}\left(f_{n}\right), \cdots, A^{\varepsilon(1)}\left(f_{1}\right)\right\}$ there are same number of annihilators and creators, then there exists a constant $c$ such that

$$
A^{\varepsilon(n)}\left(f_{n}\right) \cdots A^{\varepsilon(1)}\left(f_{1}\right) \Phi=c \Phi
$$

ii) if among $\left\{A^{\varepsilon(n)}\left(f_{n}\right), \cdots, A^{\varepsilon(1)}\left(f_{1}\right)\right\}$ there are more annihilators than creators, then

$$
A^{\varepsilon(n)}\left(f_{n}\right) \cdots A^{\varepsilon(1)}\left(f_{1}\right) \Phi=0
$$

iii) if the cardinality of the set $\{i: \varepsilon(i)= \pm 1\}$ is odd, then

$$
A^{\varepsilon(n)}\left(f_{n}\right) A^{\varepsilon(n-1)}\left(f_{n-1}\right) \cdots A^{\varepsilon(1)}\left(f_{1}\right) \Phi=0
$$

iv) if either $\varepsilon(1) \in\{0,-1\}$ or $\varepsilon(n) \in\{1,0\}$, the scalar product

$$
\left\langle A^{\varepsilon(n)}\left(f_{n}\right) A^{\varepsilon(n-1)}\left(f_{n-1}\right) \cdots A^{\varepsilon(1)}\left(f_{1}\right)\right\rangle
$$

is equal to zero.

Proof. It clearly follows from the definitions.

Definition 3.1 For $n \in \mathbb{N}$ and $\varepsilon=(\varepsilon(n), \cdots, \varepsilon(1)) \in\{-1,0,1\}^{n}$ we define the depth function (of the string $\varepsilon$ ) $d_{\varepsilon}:\{1, \ldots, n\} \rightarrow\{0, \pm 1, \ldots, \pm n\}$ by

$$
\begin{gathered}
d_{\varepsilon}(j)=\sum_{k=1}^{j} \varepsilon(k) \\
=|\{\varepsilon(k): \varepsilon(k)=1 ; k<j\}|-|\{\varepsilon(k): \varepsilon(k)=-1 ; k<j\}|
\end{gathered}
$$

Thus $d_{\varepsilon}(j)$ gives the relative number of creators (annihilators, if negative) in the product $A^{\varepsilon(n)} \cdots A^{\varepsilon(1)}$ which are at the right of $A^{\varepsilon(j)}$ or, equivalently, the number of pairs which contain $j$ in their "interior".

Definition $3.2\{-1,0,1\}_{+}^{n}$ is defined as the totality of all $\{-1,0,1\}^{n}$ satisfying the following conditions:

i) $\sum_{k=1}^{n} \varepsilon(k)=d_{\varepsilon}(n)=0$; 
ii) $\varepsilon(1)=1$ and $\varepsilon(n)=-1$;

iii) for all $i=1, \ldots, n, d_{\varepsilon}(i) \geq 0$.

Lemma 3.2 For any $n \in \mathbb{N},\left\{f_{k}\right\}_{k=1}^{n} \subset H$ and $\varepsilon \in\{-1,0,1\}^{n}$, the scalar product

$$
\left\langle A^{\varepsilon(n)}\left(f_{n}\right) A^{\varepsilon(n-1)}\left(f_{n-1}\right) \cdots A^{\varepsilon(1)}\left(f_{1}\right)\right\rangle
$$

can be nonzero only if $\varepsilon \in\{-1,0,1\}_{+}^{n}$.

Remark 3.1 If we restrict our consideration only to creation and annihilation operators, the analogue of the set $\{-1,0,1\}_{+}^{n}$, is denoted by $\{-1,1\}_{+}^{n}$.

Condition i), which can be realized only if $n$ is even, means that within the set

$$
\left\{A^{\varepsilon(n)}\left(f_{n}\right), A^{\varepsilon(n-1)}\left(f_{n-1}\right), \cdots, A^{\varepsilon(1)}\left(f_{1}\right)\right\}
$$

the number of creators is equal to the number of annihilators. Condition iii) means that for any $i$, in the set $\left\{A^{\varepsilon(i)}\left(f_{i}\right), A^{\varepsilon(i-1)}\left(f_{i-1}\right), \cdots, A^{\varepsilon(1)}\left(f_{1}\right)\right\}$ there are more creators than annihilators. As a consequence, $\varepsilon(1)$ must be equal to 1. Moreover, since one must verify contemporarily both

$$
|\{h: h \leq n-1, \varepsilon(h)=1\}| \geq|\{h: h \leq n-1, \varepsilon(h)=-1\}|
$$

and

$$
\sum_{k=1}^{n} \varepsilon(k)=0,
$$

$\varepsilon(n)$ has to be equal to -1 .

When one considers not only creation and annihilation, but also number operators, the cardinality of number operators is arbitrary, so that $n$ is not necessarily even.

Proof of Lemma 3.2. Condition ii) is clear. iii) follows from the identity:

$$
A^{\varepsilon(i)}\left(f_{i}\right) \ldots A^{\varepsilon(1)}\left(f_{1}\right) \Phi \in H_{\sum_{j=1}^{i} \varepsilon(j)} ; \quad i \in\{2, \ldots, n\}
$$

with the convention that

$$
H_{\nu}=0, \quad \text { for } \quad \nu<0
$$


If i) does not holds, then either the number of creators from the left is less than that of the annihilators or the converse is true.In the former case (3.3) is zero because of iii). In the latter the adjoint of (3.3) is

$$
\left\langle A^{\varepsilon^{\prime}(1)}\left(\hat{f}_{1}\right) \ldots A^{\varepsilon^{\prime}(n)}\left(\hat{f}_{n}\right)\right\rangle
$$

where

$$
\begin{gathered}
\varepsilon^{\prime}(j)=\left\{\begin{array}{r}
1-\varepsilon(j), \quad \text { if } \varepsilon(j)= \pm 1 \\
\varepsilon(j), \quad \text { if } \varepsilon(j)=0
\end{array}\right. \\
\hat{f}_{j}=\left\{\begin{array}{rr}
f_{j}, & \text { if } \quad \varepsilon(j)= \pm 1 \\
\bar{f}_{j}, & \text { if } \quad \varepsilon(j)=0
\end{array}\right.
\end{gathered}
$$

and this is zero because of iii).

Definition 3.3 For any pair partition $\left\{\left(i_{p}, j_{p}\right)\right\}_{p=1}^{n}$ of the set $\{1,2, \cdots, 2 n\}$, $\left\{\left(a_{i_{p}}, a_{j_{p}}\right)\right\}_{p=1}^{n}$ will be called a pair partition of the set $\left\{a_{1}, a_{2}, \cdots, a_{2 n}\right\}$. $\left\{\left(a_{i_{p}}, a_{j_{p}}\right)\right\}_{p=1}^{n}$ is called non-crossing if $\left\{\left(i_{p}, j_{p}\right)\right\}_{p=1}^{n}$ is non-crossing.

We shall adopt the convention that, for any pair partition (non-crossing or crossing $)\left\{\left(a_{i_{p}}, a_{j_{p}}\right)\right\}_{p=1}^{n}$, we have $i_{n}<\cdots<i_{1}$ and $i_{p}>j_{p}$ for all $p=$ $1,2, \cdots, n$.

We have shown that for any $\varepsilon \in\{-1,0,1\}_{+}^{n}$, the cardinality of the set $\{i: \varepsilon(i)= \pm 1\}$ must be even and we shall denote this set by $\left\{i_{1}, i_{2}, \cdots, i_{2 N}\right\}$ with the order $i_{2 N}<\cdots<i_{1}$. Define

$$
\varepsilon^{\prime}(h):=\varepsilon\left(i_{h}\right), \quad \forall h=1,2, \cdots, 2 N
$$

then $\varepsilon^{\prime} \in\{-1,1\}_{+}^{2 N}$ and, as proved in [1], $\varepsilon^{\prime}$ determines a unique non-crossing pair partition of the set $\{1,2, \cdots, 2 N\}$, hence a non-crossing pair partition of $\left\{i_{1}, i_{2}, \cdots, i_{2 N}\right\}$. In the following this pair partition will be called the noncrossing pair partition determined by $\varepsilon$. It is clear that any $\varepsilon \in\{-1,0,1\}_{+}^{n}$ determines exactly one $\varepsilon^{\prime}$ and therefore exactly one pair partition.

Lemma 3.3 In the notation (2.4), one has:

$$
\left\langle\Phi,\left(A(f)+A^{+}(f)+\Lambda_{\alpha}\right)^{m} \Phi\right\rangle=\sum_{\varepsilon \in\{-1,0,1\}_{+}^{m}}\left\langle\Phi, A^{\varepsilon(m)} \ldots A^{\varepsilon(1)} \Phi\right\rangle
$$




$$
=\sum_{\substack{0 \leq j \leq m-2 \\ m-j \in 2 \mathbb{N}}} \sum_{\substack{i_{j}, \ldots, i_{1}=2 \\ i_{j}<\ldots<i_{1}}}^{m-1} \sum_{\substack{\varepsilon \in\{-1,0,1\}^{m, j} \\ \varepsilon\left(i_{s}\right)=0}} \prod_{s=1}^{j} \alpha_{d_{\varepsilon}(s)=1,2, \ldots, j}\left\langle\Phi, \prod_{\substack{1 \leq h \leq m h \notin\left\{i_{s}\right\}_{s=1}^{j} \\ 1}} A^{\varepsilon(h)}(f) \Phi\right\rangle
$$

where, for any $j \in\{h: h=0,1, \cdots, m-2\}$ such that $m-j$ is even and for any $s=1, \ldots, j$, we define

$$
\{-1,0,1\}_{+}^{m, j}:=\left\{\varepsilon \in\{-1,0,1\}_{+}^{m} ; \sharp\{h: h=1,2, \cdots, m, \varepsilon(h)=0\}=j\right\}
$$

Proof. Expanding the power $\left(A(f)+A^{+}(f)+\Lambda_{\alpha}\right)^{m}$, we find that

$$
\left\langle\Phi,\left(A(f)+A^{+}(f)+\Lambda_{\alpha}\right)^{m} \Phi\right\rangle=\sum_{\varepsilon \in\{-1,0,1\}^{m}}\left\langle\Phi, A^{\varepsilon(m)} \cdots A^{\varepsilon(1)} \Phi\right\rangle
$$

By Lemma 3.2, for any $\varepsilon \in\{-1,0,1\}^{m} \backslash\{-1,0,1\}_{+}^{m}$, the scalar product $\left\langle\Phi, A^{\varepsilon(m)} \cdots A^{\varepsilon(1)} \Phi\right\rangle$ is equal to zero and so one gets the first identity in (3.4).

For any $\varepsilon \in\{-1,0,1\}^{m}$, denoting

$$
j:=|\{h: h=1,2, \cdots, m, \varepsilon(h)=0\}|
$$

we see that $j$ can take values in the set $\{0,1,2, \cdots, m\}$, that is,

$$
\sum_{\varepsilon \in\{-1,0,1\}^{m}}\left\langle\Phi, A^{\varepsilon(m)} \cdots A^{\varepsilon(1)} \Phi\right\rangle=\sum_{j=0}^{m} \sum_{\varepsilon \in\{-1,0,1\}_{+}^{m, j}}\left\langle\Phi, A^{\varepsilon(m)} \cdots A^{\varepsilon(1)} \Phi\right\rangle
$$

If $j=m$, i.e if $\varepsilon(i)=0$ for any $i=1,2, \cdots, m$, by the definition $\varepsilon \notin$ $\{-1,0,1\}_{+}^{m}$. If $j=m-1$, then among all operators $\left\{A^{\varepsilon(m)}, \cdots, A^{\varepsilon(1)}\right\}$ there is exactly one creator or annihilator and all others are number operator, so $\sum_{k=1}^{m} \varepsilon(k)= \pm 1$ and by the definition $\varepsilon \notin\{-1,0,1\}_{+}^{m}$. In conclusion, as $\varepsilon$ runs over $\{-1,0,1\}_{+}^{m}$, the index $j$ should run over the set $\{0,1,2, \cdots, m-2\}$. Moreover, since in the set $\left\{A^{\varepsilon(1)}, \cdots, A^{\varepsilon(m)}\right\}$, the number of creation and annihilation operators is $m-j$, the scalar product $\left\langle\Phi, A^{\varepsilon(m)} \cdots A^{\varepsilon(1)} \Phi\right\rangle$ is equal to zero if $m-j$ is odd. In other words,

$$
\sum_{\varepsilon \in\{-1,0,1\}^{m}}\left\langle\Phi, A^{\varepsilon(m)} \cdots A^{\varepsilon(1)} \Phi\right\rangle=\sum_{\substack{0 \leq j \leq m-2 \\ m-j \in 2 \mathbb{N}}} \sum_{\varepsilon \in\{-1,0,1\}_{+}^{m, j}}\left\langle\Phi, A^{\varepsilon(m)} \cdots A^{\varepsilon(1)} \Phi\right\rangle
$$


For any $j=0,1, \cdots, m-2$ such that $m-j$ is even and for any $\varepsilon \in$ $\{-1,0,1\}_{+}^{m, j}$, we denote $\left\{i_{1}, \cdots, i_{j}\right\}=\{h: \varepsilon(h)=0\}$ with the order $i_{j}<$ $\cdots<i_{1}$. By the definition of $\{-1,0,1\}_{+}^{m}, 1 \notin\left\{i_{1}, \cdots, i_{j}\right\}$ and $m \notin\left\{i_{1}, \cdots, i_{j}\right\}$, i.e. $2 \leq i_{j}<\cdots<i_{1} \leq m-1$. So

$$
\begin{aligned}
& \sum_{\substack{0 \leq j \leq m-2 \\
m-j \in 2 \mathbb{N}}} \sum_{\varepsilon \in\{-1,0,1\}_{+}^{m, j}}\left\langle\Phi, A^{\varepsilon(m)} \cdots A^{\varepsilon(1)} \Phi\right\rangle \\
& =\sum_{\substack{0 \leq j \leq m-2 \\
m-j \in 2 \mathbb{N}}} \sum_{i_{j}<\ldots<i_{1} \in\{2, \ldots, m-1\}} \sum_{\varepsilon \in\{-1,0,1\}_{+}^{m, j}: \varepsilon\left(i_{s}\right)=0}\left\langle\Phi, A^{\varepsilon(m)} \cdots A^{\varepsilon(1)} \Phi\right\rangle
\end{aligned}
$$

For any $j=0,1, \cdots, m-2$ such that $m-j$ is even, for any $2 \leq i_{j}<\cdots<$ $i_{1} \leq m-1$ and for any $\varepsilon \in\{-1,0,1\}_{+}^{m, j}$ such that $\varepsilon\left(i_{s}\right)=0 \forall s=1,2, \cdots, j$, by definition, the scalar product $\left\langle\Phi, A^{\varepsilon(m)} \cdots A^{\varepsilon(1)} \Phi\right\rangle$ must have the form

$$
\begin{gathered}
\left\langle\Phi, A^{\varepsilon(m)}(f) \cdots A^{\varepsilon\left(i_{j}+1\right)}(f) \Lambda_{\alpha} A^{\varepsilon\left(i_{j}-1\right)}(f) \cdots\right. \\
\left.\cdots A^{\varepsilon\left(i_{1}+1\right)}(f) \Lambda_{\alpha} A^{\varepsilon\left(i_{1}-1\right)}(f) \cdots A^{\varepsilon(1)}(f) \Phi\right\rangle
\end{gathered}
$$

By definition, the vector $\Lambda_{\alpha} A^{\varepsilon\left(i_{1}-1\right)}(f) \cdots A^{\varepsilon(1)}(f) \Phi$ is a certain $\alpha_{n}$ multiplied by $A^{\varepsilon\left(i_{1}-1\right)}(f) \cdots A^{\varepsilon(1)}(f) \Phi$, where the number $n$ must be equal to the difference between the number of creators among

$$
\left\{A^{\varepsilon\left(i_{1}-1\right)}(f), \cdots, A^{\varepsilon(1)}(f)\right\}
$$

and that of annihilators, i.e. $d_{\varepsilon}(1)$. So we have that

$$
\Lambda_{\alpha} A^{\varepsilon\left(i_{1}-1\right)}(f) \cdots A^{\varepsilon(1)}(f) \Phi=\alpha_{d_{\varepsilon}(1)} A^{\varepsilon\left(i_{1}-1\right)}(f) \cdots A^{\varepsilon(1)}(f) \Phi
$$

By induction we prove, that the scalar product (3.6) is equal to

$$
\begin{gathered}
\prod_{s=1}^{j} \alpha_{d_{\varepsilon}(s)}\left\langle\Phi, A^{\varepsilon(m)}(f) \cdots A^{\varepsilon\left(i_{j}+1\right)}(f) A^{\varepsilon\left(i_{j}-1\right)}(f) \cdots\right. \\
\left.\cdots A^{\varepsilon\left(i_{1}+1\right)}(f) A^{\varepsilon\left(i_{1}-1\right)}(f) \cdots A^{\varepsilon(1)}(f) \Phi\right\rangle
\end{gathered}
$$

and this proves the second identity in (3.4).

Remark 3.2 In the notations of Lemma 3.3, if instead of the operator $\Lambda_{\alpha}=$ $\Lambda_{\alpha}(I)$ one considers $\Lambda_{\alpha}(\chi)$, where $\chi$ is the indicator of any measurable set including the support of $f$, the identity (3.4) remains true.

Remark 3.3 A similar version of the above result is given in [3] Theorem 5.1. where a different notation is used. 


\section{$\S 4$ 1-mode type IFS}

The simplest class of standard IFS is that for which the functions $\left(\lambda_{n}\right)_{n}$ in Definition 2.1 are constants. The sequence $\left\{\alpha_{n}\right\}_{n=1}^{\infty} \subset \mathbb{R}$ is arbitrary while condition (ii) of Definition 2.1 becomes in this case: $\left\{\lambda_{n}\right\}_{n=1}^{\infty} \subset \mathbb{R}_{+}$and $\lambda_{n}=0 \Rightarrow \lambda_{n+1}=0 \forall n$. Any pair of sequences satisfying these conditions will be called a pair of Jacobi sequences.

In the notation (2.4) this class is characterized by the following relations:

$$
\begin{gathered}
A_{f} A_{g}^{+}=\omega_{\Lambda+1}\langle f, g\rangle \text { for any } f, g \in H \\
A_{f} \Phi=0 \text { for any } f \in H \\
\omega_{\Lambda}=\sum_{n=0}^{\infty} \omega_{n} P_{n}
\end{gathered}
$$

$P_{n}$ denotes the projection on the $n$-particle space in the decomposition (2.1) and

$$
\omega_{n}:=\frac{\lambda_{n}}{\lambda_{n-1}}
$$

This means that all the mixed moments of $A_{f}^{\varepsilon}(\varepsilon \in\{-1,0,1\})$ are uniquely determined by $f$ and by the basic relation

$$
\alpha_{\Lambda} A^{\varepsilon(n)}\left(f_{n}\right) \cdots A^{\varepsilon(1)}\left(f_{1}\right) \Phi=\alpha_{d_{\varepsilon}(n)} A^{\varepsilon(n)}\left(f_{n}\right) \cdots A^{\varepsilon(1)}\left(f_{1}\right) \Phi
$$

where $d_{\varepsilon}$ is the depth function defined in 3.2 .

Lemma 4.1 If the functions $\left\{\lambda_{n}\right\}_{n=1}^{\infty}$ are constants then for any $m \in \mathbb{N}$ and for any $\varepsilon \in\{-1,1\}^{m}$, the scalar product

$$
\left\langle\Phi, A^{\varepsilon(m)}(f) \cdots A^{\varepsilon(1)}(f) \Phi\right\rangle
$$

has the form

$$
\|f\|^{m} \cdot C\left(\varepsilon ;\left\{\lambda_{n}\right\}_{n=1}^{\infty}\right)
$$

where $\|\cdot\|$ is the norm in $L^{2}(X, \mu), C\left(\varepsilon ;\left\{\lambda_{n}\right\}_{n=1}^{\infty}\right)$ is a constant uniquely determined by $\varepsilon$ and $\left\{\lambda_{n}\right\}_{n=1}^{m}$. Moreover it is equal to zero if either $m$ is odd or $m$ is even and $\varepsilon \in\{-1,1\}^{m} \backslash\{-1,1\}_{+}^{m}$. 
Proof. If $m$ is odd, the conclusion is trivial and so we consider only the case $m=2 N$ for some $N \in \mathbb{N}$. As proved in Lemma 3.2. one knows that $\left\langle\Phi, A^{\varepsilon(2 N)}(f) \cdots A^{\varepsilon(1)}(f) \Phi\right\rangle=0$ if $\varepsilon \in\{-1,1\}^{2 N} \backslash\{-1,1\}_{+}^{2 N}$. In the case of $N=1$ and $\varepsilon \in\{-1,1\}_{+}^{2}$

$$
\left\langle\Phi, A^{\varepsilon(2)}(f) A^{\varepsilon(1)}(f) \Phi\right\rangle=\left\langle\Phi, A(f) A^{+}(f) \Phi\right\rangle=\lambda_{1}\|f\|^{2}
$$

We suppose the statement is true for any $k \leq N$ and prove it for $k=N+1$. For any $\varepsilon \in\{-1,1\}_{+}^{2(N+1)}$, by denoting

$$
h:=\min \{k \in\{1, \ldots, 2 N+2\}, \varepsilon(k)=-1\}
$$

we know that, just by definition, $h \neq 1$ and $\varepsilon(h-1)=\cdots=\varepsilon(1)=1$. Moreover since

$$
A(f) A^{+}\left(f_{n}\right) \cdots A^{+}\left(f_{1}\right) \Phi=\omega_{n}\left\langle f, f_{n}\right\rangle A^{+}\left(f_{n-1}\right) \cdots A^{+}\left(f_{1}\right) \Phi
$$

we have that

$$
\begin{gathered}
\left\langle\Phi, A^{\varepsilon(2(N+1))}(f) \cdots A^{\varepsilon(1)}(f) \Phi\right\rangle \\
=\langle f, f\rangle \omega_{d_{\varepsilon}(h)}\left\langle\Phi, A^{\varepsilon(2(N+1))}(f) \cdots A^{\varepsilon(h+2)}(f) A^{\varepsilon(h+1)}(f) \cdots A^{\varepsilon(1)}(f) \Phi\right\rangle
\end{gathered}
$$

The proof follows now by induction .

Corollary 4.1 For any $f, g \in H$ the moments of $A(f)+A^{+}(f)$ and of $A(g)+A^{+}(g)$ are the same if and only if $\|f\|=\|g\|$.

Proof. Our conclusion is easy to see as follows

$$
\begin{gathered}
\left\langle\Phi,\left(A(f)+A^{+}(f)\right)^{m} \Phi\right\rangle=\sum_{\varepsilon \in\{-1,1\}_{+}^{m}}\left\langle\Phi, A^{\varepsilon(m)}(f) \ldots A^{\varepsilon(1)}(f) \Phi\right\rangle \\
=\|f\|^{m} \sum_{\varepsilon \in\{-1,1\}_{+}^{m}} C\left(\varepsilon ;\left\{\lambda_{n}\right\}_{n=1}^{\infty}\right) \\
=\|g\|^{m} \sum_{\varepsilon \in\{-1,1\}_{+}^{m}} C\left(\varepsilon ;\left\{\lambda_{n}\right\}_{n=1}^{\infty}\right)=\left\langle\Phi,\left(A(g)+A^{+}(g)\right)^{m} \Phi\right\rangle
\end{gathered}
$$


Corollary 4.2 For any $f, g \in H$ and $\chi_{f}, \chi_{g}$ two indicators such that $\chi_{f}$ (resp. $\chi_{g}$ ) is the indicator of a measurable set including the support of $f$ (resp. $g$ ), the moments of $A(f)+A^{+}(f)+\Lambda_{\alpha}\left(\chi_{f}\right)$ and $A(g)+A^{+}(g)+\Lambda_{\alpha}\left(\chi_{g}\right)$ are the same if and only if $\|f\|=\|g\|$.

Proof. For any $m \in \mathbb{N}$

$$
\begin{aligned}
& \left\langle\Phi,\left(A(f)+A^{+}(f)+\Lambda_{\alpha}\left(\chi_{f}\right)\right)^{m} \Phi\right\rangle \\
& =\sum_{\substack{0 \leq j \leq m-2 \\
m-j \in 2 \mathbb{N}}} \sum_{\substack{i_{j}, \ldots, i_{1}=2 \\
i_{j}<\cdots<i_{1}}}^{m-1} \sum_{\substack{\varepsilon \in\{-1,0,1\}_{+}^{m, j} \\
\varepsilon\left(i_{s}\right)=0 \\
\forall s=1,2, \ldots, j}} \alpha_{d_{\varepsilon}(s)}\left\langle\Phi, \prod_{\substack{1 \leq h \leq m \\
h \notin\left\{i_{s}\right\}_{s=1}^{j}}} A^{\varepsilon(h)}(f) \Phi\right\rangle
\end{aligned}
$$

and

$$
\begin{aligned}
& \left\langle\Phi,\left(A(g)+A^{+}(g)+\Lambda_{\alpha}\left(\chi_{g}\right)\right)^{m} \Phi\right\rangle \\
& =\sum_{\substack{0 \leq j \leq m-2 \\
m-j \in 2 \mathbb{N}}} \sum_{\substack{i_{j}, \ldots, i_{1}=2 \\
i_{j}<\cdots<i_{1}}}^{m-1} \sum_{\substack{\varepsilon \in\{-1,0,1\}_{+}^{m, j} \\
\varepsilon\left(i_{s}\right)=0 \quad \forall s=1,2, \ldots, j}} \prod_{s=1}^{j} \alpha_{d_{\varepsilon}(s)}\left\langle\Phi, \prod_{\substack{1 \leq h \leq m \\
h \notin\left\{i_{s}\right\}_{s=1}^{j}}} A^{\varepsilon(h)}(g) \Phi\right\rangle
\end{aligned}
$$

For any $0 \leq j \leq m-2$ such that $m-j \in 2 \mathbb{N}$, for any $2 \leq i_{j}<\ldots<i_{1} \leq m-1$ and for any $\varepsilon \in\{-1,0,1\}_{+}^{m, j}$ such that $\varepsilon\left(i_{s}\right)=0 \forall s=1,2, \cdots, j$, it follows from Lemma 4.1 that

$$
\left\langle\Phi, \prod_{\substack{1 \leq h \leq m \\ h \notin\left\{i_{s}\right\}_{s=1}^{j}}} A^{\varepsilon(h)}(f) \Phi\right\rangle=\left\langle\Phi, \prod_{\substack{1 \leq h \leq m \\ h \notin\{i s\}_{s=1}^{j}}} A^{\varepsilon(h)}(g) \Phi\right\rangle
$$

if and only if $\|f\|=\|g\|$. Hence

$$
\left\langle\Phi,\left(A(f)+A^{+}(f)+\Lambda_{\alpha}\left(\chi_{f}\right)\right)^{m} \Phi\right\rangle=\left\langle\Phi,\left(A(g)+A^{+}(g)+\Lambda_{\alpha}\left(\chi_{g}\right)\right)^{m} \Phi\right\rangle
$$

if and only if $\|f\|=\|g\|$.

Remark 4.1 Both Corollaries 4.1 and 4.2 strongly depend on the fact that the $\left(\lambda_{n}\right)$ are constant. There are many standard IFS in which the distribution of $A(f)+A^{+}(f)+\Lambda_{\alpha}\left(\chi_{f}\right)$ (even $A(f)+A^{+}(f)$ ) depends not only on $\|f\|$ but also on $f$ itself. For example, if we take $H:=\mathbf{L}^{2}([0,1])$ and $\lambda_{n}\left(x_{1}, \cdots, x_{n}\right):=x_{2} x_{3}^{2} \cdots x_{n}^{n-1}$ for any $n$, then the distribution of $A\left(\chi_{[0,1]}\right)+$ $A^{+}\left(\chi_{[0,1]}\right)$ is the arcsine law but $A\left(\sqrt{2} \chi_{[0,1 / 2]}\right)+A^{+}\left(\sqrt{2} \chi_{[0,1 / 2]}\right)$ has a different distribution. For more example see [2, 18, 19] and references therein. 
Corollary 4.3 Let $\|f\|=1$. Then the polynomial distribution of $A_{f}^{+}+A_{f}+$ $\alpha_{N}$ is the (polynomially unique) probability measure with Jacobi parameters $\left(\omega_{n}\right),\left(\alpha_{n}\right)$.

Proof. Let $\left\{a^{ \pm}, \mathcal{H}_{1}, \Phi_{1}\right\}$ denote the 1-mode interacting Fock space with Jacobi parameters $\left(\omega_{n}\right),\left(\alpha_{n}\right)$. The identity (4.6) and 4.4) imply that

$$
A_{f} A_{f}^{+}=\frac{\lambda_{N}}{\lambda_{N-1}}=\omega_{N}
$$

Since $A_{f} \Phi=0$, it follows that the map

$$
a^{+n} \Phi_{1} \mapsto A_{f}^{+n} \Phi
$$

extends to a unitary isomorphism $U$, from $\mathcal{H}_{1}$ to the closed subspace generated by the vectors $\left\{A_{f}^{+n} \Phi: n \in \mathbb{N}\right\}$. By construction $U$ satisfies

$$
\begin{gathered}
U a^{+}=A_{f}^{+} U \\
U \Phi_{1}=\Phi
\end{gathered}
$$

and this implies the statement.

Lemma 4.2 If the $\left\{\lambda_{n}\right\}_{n=1}^{\infty}$ are constant, then for any $N \in \mathbb{N}$, for any $\varepsilon \in\{-1,1\}_{+}^{2 N}$ and for any $\left\{f_{1}, \cdots, f_{2 N}\right\} \subset H$, with the convention $\lambda_{0}:=1$, one has:

$$
\left\langle\Phi, A^{\varepsilon(2 N)}\left(f_{2 N}\right) \cdots A^{\varepsilon(1)}\left(f_{1}\right) \Phi\right\rangle=\prod_{k=1}^{N}\left\langle f_{l_{k}}, f_{r_{k}}\right\rangle \omega_{d_{\varepsilon}\left(r_{k}\right)}
$$

where $\left\{l_{k}, r_{k}\right\}_{k=1}^{N}$ is the unique non-crossing pair partition determined by $\varepsilon \in\{-1,1\}_{+}^{2 N}$.

Proof. If $N=1,4.8$ is clear. Suppose that it holds for $N=n$. Then, for $N=n+1$ one has:

$$
\begin{gathered}
\left\langle\Phi, A^{\varepsilon(2 n+2)}\left(f_{2 n+2}\right) \cdots A^{\varepsilon(1)}\left(f_{1}\right) \Phi\right\rangle \\
=\left\langle\Phi, A^{\varepsilon(2 n+2)}\left(f_{2 n+2}\right) \cdots A^{\varepsilon\left(l_{n+1}\right)}\left(f_{l_{n+1}}\right) A^{\varepsilon\left(r_{n+1}\right)}\left(f_{r_{n+1}}\right) \cdots A^{\varepsilon(1)}\left(f_{1}\right) \Phi\right\rangle .
\end{gathered}
$$


By the non-crossing principle $r_{n+1}=l_{n+1}-1$ and $\varepsilon(h)=1$ for any $h \leq r_{n+1}$. So the quantity above is equal to

$$
\begin{aligned}
& \left\langle A^{\varepsilon(2 n+2)}\left(f_{2 n+2}\right) \cdots A^{\varepsilon\left(l_{n+1}\right)+1}\left(f_{l_{n+1}+1}\right) A\left(f_{l_{n+1}+1}\right) A^{+}\left(f_{r_{n+1}}\right) A^{+}\left(f_{r_{n+1}-1}\right) \cdots A^{+}\left(f_{1}\right)\right\rangle \\
& =\left\langle f_{l_{n+1}}, f_{r_{n+1}}\right\rangle \omega_{d_{\varepsilon}\left(l_{n+1}\right)} \times \\
& \times\left\langle A^{\varepsilon(2 n+2)}\left(f_{2 n+2}\right) \cdots A^{\varepsilon\left(l_{n+1}+1\right)}\left(f_{l_{n+1}+1}\right) A^{+}\left(f_{r_{n+1}-1}\right) \cdots A^{+}\left(f_{1}\right)\right\rangle \\
& =\left\langle f_{l_{n+1}}, f_{r_{n+1}}\right\rangle \omega_{d_{\varepsilon}\left(l_{n+1}\right)} \times \\
& \quad \times\left\langle A^{\varepsilon(2 n+2)}\left(f_{2 n+2}\right) \cdots A^{\varepsilon\left(l_{n+1}+1\right)}\left(f_{l_{n+1}+1}\right) A^{\varepsilon\left(r_{n+1}-1\right)}\left(f_{r_{n+1}-1}\right) \cdots A^{+}\left(f_{1}\right)\right\rangle
\end{aligned}
$$

(4.8) now follows from the induction assumption.

\section{$\S 5$ Central limit theorem}

The main goal in this section is to show our central limit theorem. For any pair of Jacobi sequences $\left\{\alpha_{n}\right\}_{n=1}^{\infty} \subset \mathbb{R},\left\{\lambda_{n}\right\}_{n=1}^{\infty} \subset \mathbb{R}_{+}$, in the notations 2.2), 2.3 we consider the following operators on the 1-MT-IFS $\Gamma\left(\mathbf{L}^{2}\left(\mathbb{R}_{+}\right),\left\{\lambda_{n}\right\}_{n=1}^{\infty}\right)$ :

$$
A_{k}:=A\left(\chi_{[k, k+1)}\right) ; A_{k}^{+}:=A^{+}\left(\chi_{[k, k+1)}\right), \Lambda_{k}:=\Lambda_{\alpha}, \quad k=0,1, \ldots
$$

and for any bounded sequence $\left\{c_{k}\right\}_{k=1}^{\infty} \subset \mathbb{R}$ we consider

$$
Q_{k}=A_{k}+A_{k}^{+}+c_{k} \Lambda_{k}, \quad k=0,1,2, \ldots
$$

and $c_{0}:=1$.

Lemma 5.1 The family $\left\{A_{k}, A_{k}^{+}\right\}_{k=1}^{\infty}$ satisfies the singleton condition with respect to the state $\langle\Phi, \cdot \Phi\rangle$.

Proof. Clear from 4.8.

Lemma 5.2 (Uniform boundedness of the mixed moments). For any $N \in \mathbb{N}$, for any $\left\{k_{1}, \ldots, k_{m}\right\} \subset \mathbb{N}$ and for any $\varepsilon \in\{-1,1\}_{+}^{2 N}$

$$
\left|\left\langle A_{k_{2 N}}^{\varepsilon(2 N)} \ldots A_{k_{1}}^{\varepsilon(1)}\right\rangle\right| \leq[\lambda(N)]^{2 N}
$$


with the conventions: $\lambda_{0}:=1$ and, for any $m \in \mathbb{N}$ :

$$
\lambda(m):=\max \left\{1, \lambda_{1}, \frac{\lambda_{2}}{\lambda_{1}}, \lambda_{2}, \frac{\lambda_{3}}{\lambda_{2}}, \lambda_{3}, \cdots, \frac{\lambda_{m}}{\lambda_{m-1}}, \lambda_{m}\right\} .
$$

Proof. If $N=1,\left\langle A_{k_{2}}^{\varepsilon(2)} A_{k_{1}}^{\varepsilon(1)}\right\rangle$ is different from zero only if $\varepsilon(2)=-1$ and $\varepsilon(1)=1$. Moreover

$$
\left|\left\langle A_{k_{2}}^{\varepsilon(2)} A_{k_{1}}^{\varepsilon(1)}\right\rangle\right|=\left|\left\langle A_{k_{2}} A_{k_{1}}^{+}\right\rangle\right|=\lambda_{1}\left\langle\chi_{\left[k_{2}, k_{2}+1\right]}, \chi_{\left[k_{1}, k_{1}+1\right]}\right\rangle \leq \lambda_{1} \leq \lambda
$$

Suppose that the result is true for $N$. For any $\varepsilon \in\{-1,1\}_{+}^{2(N+1)}$, we denote $\left\{l_{h}, r_{h}\right\}_{h=1}^{N+1}$ the non-crossing pair partition determined by $\varepsilon$. We know, by the non-crossing principle, that $r_{N+1}=l_{N+1}-1, \varepsilon(h)=1$ for any $h \leq r_{N+1}$, and

$$
\begin{aligned}
& \left|\left\langle A_{k_{2 N+2}}^{\varepsilon(2 N+2)} \ldots A_{k_{1}}^{\varepsilon(1)}\right\rangle\right|=\left|\left\langle A_{k_{2 N+2}}^{\varepsilon(2 N+2)} \ldots A_{k_{l_{N+1}+1}}^{\varepsilon\left(l_{N+1}+1\right)} A_{k_{l_{N+1}}} A_{k_{r_{N+1}}}^{+} A_{k_{r_{N+1}-1}}^{+} \ldots A_{k_{1}}^{+}\right\rangle\right| \\
& =\omega_{d_{\varepsilon}\left(l_{N+1}\right)} \mid\left\langle\chi_{\left[k_{l_{N+1}}, k_{l_{N+1}}+1\right]}, \chi_{\left[k_{r_{N+1}}, k_{r_{N+1}}+1\right]}\right\rangle \times \\
& \times\left\langle A_{k_{2 N+2}}^{\varepsilon(2 N+2)} \ldots A_{k_{l_{N+1}+1}}^{\varepsilon\left(l_{N+1}+1\right)} A_{k_{r_{N+1}-1}}^{+} \ldots A_{k_{1}}^{+}\right\rangle \mid
\end{aligned}
$$

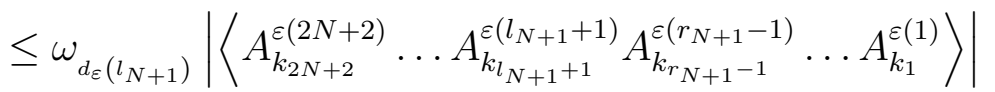

and by the induction assumption one has that

$$
\left|\left\langle A_{k_{2 N+2}}^{\varepsilon(2 N+2)} \ldots A_{k_{l_{N+1}+1}}^{\varepsilon\left(l_{N+1}+1\right)} A_{k_{r_{N+1}-1}}^{\varepsilon\left(r_{N+1}-1\right)} \ldots A_{k_{1}}^{\varepsilon(1)}\right\rangle\right| \leq[\lambda(N)]^{2 N}
$$

Therefore

$$
\left|\left\langle A_{k_{2 N+2}}^{\varepsilon(2 N+2)} \ldots A_{k_{1}}^{\varepsilon(1)}\right\rangle\right| \leq[\lambda(N)]^{2 N} \cdot \omega_{d_{\varepsilon}\left(l_{N+1}\right)}
$$

Moreover $d_{\varepsilon}\left(l_{n+1}\right)+1=d_{\varepsilon}\left(r_{n+1}\right)$ and, since $\varepsilon(h)=1$ for any $h \leq r_{N+1}$ and since $|\{h: \varepsilon(h)=1\}|=N+1$, one gets that $d_{\varepsilon}\left(l_{n+1}\right)+1=d_{\varepsilon}\left(r_{N+1}\right) \leq N+1$

. Consequently

$$
\omega_{d_{\varepsilon}\left(l_{N+1}\right)} \leq \lambda(N+1)
$$

By definition the sequence $\{\lambda(N)\}_{N=1}^{\infty}$ is increasing, hence

$$
\left|\left\langle A_{k_{2 N+2}}^{\varepsilon(2 N+2)} \ldots A_{k_{1}}^{\varepsilon(1)}\right\rangle\right| \leq[\lambda(N)]^{2 N} \cdot \omega_{d_{\varepsilon}\left(l_{N+1}\right)} \leq \lambda(N+1)^{2(N+1)}
$$


Theorem 5.1 Let $\mu$ be a mean-zero probability measure on $(\mathbb{R}, \mathcal{B})$ with moments of any order and with Jacobi coefficients given by $\left\{\omega_{n}\right\}_{n},\left\{\alpha_{n}\right\}_{n}$. Denote $\Gamma\left(\mathbf{L}^{2}\left(\mathbb{R}_{+}\right),\left\{\lambda_{n}\right\}_{n}\right)$ the 1-mode type free IFS with $\omega_{n}=\frac{\lambda_{n}}{\lambda_{n-1}}$ for any $n$ and consider the operators $\left\{A_{k}, A_{k}^{+}, \Lambda_{k}\right\}_{k=0}^{\infty}$ defined by (5.1), (5.2) on $\Gamma\left(\mathbf{L}^{2}\left(\mathbb{R}_{+}\right),\left\{\lambda_{n}\right\}_{n}\right)$, where $\left\{c_{k}\right\}_{k=1}^{\infty} \subset \mathbb{R}$ is a bounded sequence which satisfies the condition

$$
\frac{1}{\sqrt{N}} \sum_{k=1}^{N} c_{k} \rightarrow 1
$$

(e.g. $\quad c_{k}=\sqrt{k}-\sqrt{k-1}$ for any $k$ ) if the sequence $\left(\alpha_{n}\right)$ does not vanish identically.

Then

i) for any $k=0,1,2 \cdots$, the distribution of $A_{k}+A_{k}^{+}+\Lambda_{k}$ with respect to the state $\langle\Phi, \cdot \Phi\rangle$ is exactly the measure $\mu$;

ii) the operator quantum stochastic process

$$
\left\{\frac{1}{\sqrt{N}} \sum_{k=1}^{N} A_{k}, \frac{1}{\sqrt{N}} \sum_{k=1}^{N} A_{k}^{+}, \frac{1}{\sqrt{N}} \sum_{k=1}^{N} c_{k} \Lambda_{k}\right\}_{k=1}^{\infty}
$$

converges in the sense of the mixed moments for $N \rightarrow \infty$, to

$$
\left\{A_{0}, A_{0}^{+}, \Lambda_{0}\right\}
$$

Proof. i) follows from Corollary 4.2 . Corollary 4.3 and [3. To prove ii) we first deal with the symmetric case i.e. when the sequence $\left(\alpha_{n}\right)$, hence $\left(\Lambda_{k}\right)$, identically vanishes and the quantum operator process is reduced to $\left\{(1 / \sqrt{N}) \sum_{k=1}^{N} A_{k}^{ \pm}\right\}$. Our goal consists in showing that for any $m \geq 1$ and for any $\varepsilon=(\varepsilon(1), \cdots, \varepsilon(m)) \in\{-1,+1\}^{m}$

$$
\lim _{N \rightarrow \infty} \frac{1}{N^{\frac{m}{2}}} \sum_{1 \leq \beta_{1}, \ldots, \beta_{m} \leq N}\left\langle A_{\beta_{m}}^{\varepsilon(m)} \cdots A_{\beta_{1}}^{\varepsilon(1)}\right\rangle=\left\langle A_{0}^{\varepsilon(m)} \cdots A_{0}^{\varepsilon(1)}\right\rangle
$$

The operator process $\left\{A_{k}, A_{k}^{+}\right\}_{k=0}^{\infty}$ satisfies the singleton condition and the boundedness of the mixed moments as shown in Lemma 5.1 and Lemma 5.2 respectively. Hence from [4], Lemma 2.4 it follows that the limit (5.5) is nonzero only if $m=2 p$ and, in this case, the left hand side of (5.5) is equal to:

$$
\lim _{N \rightarrow \infty} \frac{1}{N^{p}} \sum_{\substack{\left.\beta:\{1, \ldots, 2 p\} \rightarrow\{1, \ldots, N\} \\ \text { Range }(\beta)=p, \mid \beta^{-1}(\beta, j)\right) \mid=2 \forall j=1, \ldots, 2 p}}\left\langle A_{\beta_{m}}^{\varepsilon(2 p)} \cdots A_{\beta_{1}}^{\varepsilon(1)}\right\rangle
$$


where $|\cdot|$ denotes cardinality. Each map $\beta$ in the above summation induces a pair partition of the set $\{1,2, \ldots, 2 p\}$. We denote by P.P. $(2 p)$ the set of all these partitions and use the notations

$$
\beta^{-1}(\beta(j))=:\left\{l_{j}, r_{j}\right\}, \quad l_{j}>r_{j}, \quad j=1, \ldots, p
$$

Moreover we know from Lemma 3.1 that a term in the sum $(5.6)$ can be nonzero only if $\varepsilon \in\{-1,+1\}_{+}^{2 p}$. In particular only non crossing pair partitions may give nonzero contributions. Hence the quantity (5.6) can be rewritten as

$$
\lim _{N \rightarrow \infty} \frac{1}{N^{p}} \sum_{\left\{l_{j}, r_{j}\right\}_{j=1}^{p} \in N . C . P . P .(2 p)}\left\langle A_{\beta_{2 p}} \cdots A_{\beta_{l_{j}}}^{\varepsilon\left(l_{j}\right)} \cdots A_{\beta_{r_{j}}}^{\varepsilon\left(r_{j}\right)} \cdots A_{\beta_{1}}^{+}\right\rangle,
$$

where N.C.P.P. $(2 p)$ denotes the set of all non crossing pair partitions of $\{1,2, \ldots, 2 p\}$ and the first operator on the left is an annihilator (and corresponds to an $l_{j}$-index) while the first operator on the right is a creator ( and corresponds to an $r_{j}$-index). Let $\beta_{l_{1}}$ be the index of the first annihilator from the right in (5.7). Hence the operator with index $\beta_{l_{1}}-1$ is a creator. If $\beta_{l_{1}}-1 \neq \beta_{r_{1}}$, then one has

$$
A_{\beta_{l_{1}}} A_{\beta_{l_{1}}-1}^{+}=0
$$

because we are dealing with disjoint intervals. Therefore the nonzero contributions can come only from those pairs satisfying $\beta_{l_{1}}-1=\beta_{r_{1}}$. Using (4.7) and the depth function $(3.2),(5.7)$ becomes

$$
\omega_{d_{\varepsilon}\left(l_{1}\right)} \lim _{N \rightarrow \infty} \frac{1}{N^{p}} \sum_{\left\{l_{j}, r_{j}\right\}_{j=1}^{p} \in N . C . P . P .(2 p)}\left\langle A_{\beta_{2(p-1)}} \cdots \widehat{A_{\beta_{l_{1}}}^{\varepsilon\left(l_{1}\right)}} \widehat{A_{\beta_{r_{1}}}^{\varepsilon\left(r_{1}\right)}} \cdots A_{\beta_{1}}^{+}\right\rangle
$$

Iterating the same procedure for any $l_{j}(j=1, \ldots, p)$ (5.7) takes the form

$$
\prod_{j=1}^{p} \omega_{d_{\varepsilon}\left(l_{j}\right)} \lim _{N \rightarrow \infty} \frac{1}{N^{p}} \sum_{\left\{l_{j}, r_{j}\right\}_{j=1}^{p} \in N . C . P . P .(2 p)}
$$

Now we observe that, as a set:

$$
\{1, \ldots, 2 p\}=\left\{l_{1}, r_{1}, \ldots, l_{p}, r_{p}\right\}
$$


so any map of the sum in (5.8) can be written in the following way:

$$
\begin{gathered}
\beta:\left\{l_{1}, r_{1}, \ldots, l_{p}, r_{p}\right\} \rightarrow\{1, \ldots, N\}, \mid \text { Range } \beta \mid=p, \\
\beta^{-1}(\beta(j))=\left\{l_{j}, r_{j}\right\}, \quad j=1, \ldots, p
\end{gathered}
$$

Moreover, since any $\varepsilon \in\{-1,+1\}_{+}^{2 p}$ determines exactly one non-crossing pair partition of $\{1, \ldots, 2 p\}$ and $l_{p}<\cdots<l_{1}$, then, for any $j=1, \ldots, p, \beta_{r_{j}}$ is uniquely determined once one knows $\beta_{l_{j}}$. So to assign $\beta$ is equivalent to assign an injective map

$$
\beta:\left\{l_{1}, \ldots, l_{p}\right\} \rightarrow\{1, \ldots, N\}
$$

Therefore (5.8) is equal to

$$
\prod_{j=1}^{p} \omega_{d_{\varepsilon}\left(l_{j}\right)} \lim _{N \rightarrow \infty} \frac{1}{N^{p}} \mid\left\{\beta:\left\{l_{1}, \ldots, l_{p}\right\} \rightarrow\{1, \ldots, N\}: \beta \text { injective }\right\} \mid
$$

where $|\cdot|$ denotes cardinality. Since

$$
\mid\left\{\beta:\left\{l_{1}, \ldots, l_{p}\right\} \rightarrow\{1, \ldots, N\}: \beta \text { injective }\right\} \mid=\frac{N !}{(N-p) !}
$$

in the limit 5.9 we have

$$
\lim _{N \rightarrow \infty} \frac{1}{N^{p}} N(N-1) \cdots(N-(p+1)) \prod_{j=1}^{p} \omega_{d_{\varepsilon}\left(l_{j}\right)}=\prod_{j=1}^{p} \omega_{d_{\varepsilon}\left(l_{j}\right)}
$$

On the other hand, in the above notations,

$$
\begin{gathered}
\left\langle A_{0}^{\varepsilon(2 p)} \cdots A_{0}^{\varepsilon(1)}\right\rangle=\left\langle A_{0} \cdots A_{0}^{\varepsilon\left(l_{1}\right)} A_{0}^{\varepsilon\left(r_{1}\right)} \cdots A_{0}^{+}\right\rangle \\
=\omega_{d_{\varepsilon}\left(l_{1}\right)}\left\langle A_{0} \cdots \widehat{A_{0}^{\varepsilon\left(l_{1}\right)}} \widehat{A_{0}^{\varepsilon\left(r_{1}\right)}} \cdots A_{0}^{+}\right\rangle
\end{gathered}
$$

and iterating for any $j$, one obtains

$$
\left\langle A_{0}^{\varepsilon(2 p)} \cdots A_{0}^{\varepsilon(1)}\right\rangle=\prod_{j=1}^{p} \omega_{d_{\varepsilon}\left(l_{j}\right)}
$$

Now let us prove 5.5 in the non-symmetric case. 
In this case $\varepsilon=(\varepsilon(1), \cdots, \varepsilon(m)) \in\{-1,0,+1\}_{+}^{m}$ and $A_{\beta_{j}}^{\varepsilon(j)}=c_{\beta_{j}} \Lambda$. The proof is by induction on $m \geq 1$. If $m=1$, then (5.5) is verified because each side is identically equal to zero. Now we suppose that 5.5 is verified for any $h \leq m-1$ and prove it for $h=m$. We denote by $\mathfrak{I}_{Z}$ the set of indices in $\varepsilon$ corresponding to number operators, i.e.

$$
\mathfrak{I}_{Z}:=\{j \in\{1, \ldots, m\}: \varepsilon(j)=0\}
$$

Denote $s=\left|\mathfrak{I}_{Z}\right|$ the number of singletons, then $0 \leq s \leq m-2$, because $1, m \notin \mathfrak{I}_{Z}$ and $m-s=2 p$, because of iii) of Lemma 3.1. If $\mathfrak{I}_{Z}=\emptyset$, then the thesis follows because we find the symmetric case. If $\mathfrak{I}_{Z} \neq \emptyset$, then there exist $k=1, \ldots m$ such that $\varepsilon(k)=0$. Denoting $z_{1}=\min \left\{k \in\{1, \ldots, m\}: k \in \mathfrak{I}_{Z}\right\}$, the right hand side of (5.5) can be written as

$$
\left\langle A_{0}^{\varepsilon(m)} \cdots A_{0}^{\varepsilon\left(z_{1}\right)} \cdots A_{0}^{\varepsilon(1)}\right\rangle=\alpha_{d_{\varepsilon}\left(z_{1}\right)}\left\langle A_{0}^{\varepsilon(m)} \cdots \widehat{A_{0}^{\varepsilon\left(z_{1}\right)}} \cdots A_{0}^{\varepsilon(1)}\right\rangle
$$

The left hand side of (5.5) can be written as

$$
\begin{gathered}
\lim _{N \rightarrow \infty} \frac{1}{N^{\frac{m}{2}}} \sum_{1 \leq \beta_{1}, \ldots, \beta_{m} \leq N}\left\langle A_{\beta_{m}}^{\varepsilon(m)} \cdots A_{\beta_{z_{1}}}^{\varepsilon\left(z_{1}\right)} \cdots A_{\beta_{1}}^{\varepsilon(1)}\right\rangle \\
=\lim _{N \rightarrow \infty}\left[( \alpha _ { d _ { \varepsilon } ( z _ { 1 } ) } \frac { 1 } { \sqrt { N } } \sum _ { \beta _ { z _ { 1 } } \in F _ { N , z _ { 1 } } } c _ { \beta _ { z _ { 1 } } } ) \left(\frac { 1 } { N ^ { \frac { m - 1 } { 2 } } } \sum _ { \begin{array} { c } 
{ 1 \leq \beta _ { 1 } , \ldots , \beta _ { m } \leq N } \\
{ \beta _ { k } \neq \beta _ { z _ { 1 } } }
\end{array} } \left\langleA_{\beta_{m}}^{\varepsilon(m)} \cdots \widehat{\left.\left.\left.A_{\beta_{z_{1}}}^{\varepsilon\left(z_{1}\right)} \cdots A_{\beta_{1}}^{\varepsilon(1)}\right\rangle\right)\right]}\right.\right.\right.
\end{gathered}
$$

where $F_{N, z_{1}}$ is the subset of $\{1, \ldots, N\}$ in which $\beta_{z_{1}}$ can be chosen. $F_{N, z_{1}}=$ $\{1, \ldots, N\} \backslash\left\{\beta_{m}, \beta_{1}\right\}$ (because $\left.1, m \notin \mathfrak{I}_{Z}\right)$. Hence $\left|F_{N, z_{1}}\right|=N-2$. As a consequence $\frac{1}{\sqrt{N}} \sum_{z_{1} \in F_{N, z_{1}}} c_{\beta_{z_{1}}}$ has the same asymptotic of $\frac{1}{\sqrt{N}} \sum_{k=1}^{N} c_{k}$ for $N \rightarrow \infty$ because $\left\{c_{k}\right\}$ is a bounded sequence in $\mathbb{R}$. Moreover we notice that

$$
\sum_{\substack{1 \leq \beta_{1}, \ldots, \beta_{m} \leq N \\ \beta_{k} \neq \beta_{z_{1}}}}\left\langle A_{\beta_{m}}^{\varepsilon(m)} \cdots \widehat{A_{\beta_{z_{1}}}^{\varepsilon\left(z_{1}\right)}} \cdots A_{\beta_{1}}^{\varepsilon(1)}\right\rangle
$$


in 5.12 can be written as

$$
\sum_{1 \leq \beta_{1}^{\prime}, \ldots, \beta_{m-1}^{\prime} \leq N}\left\langle A_{\beta_{m-1}^{\prime}}^{\varepsilon^{\prime}(m-1)} \cdots A_{\beta_{1}^{\prime}}^{\varepsilon^{\prime}(1)}\right\rangle
$$

where $\varepsilon^{\prime}=\left(\varepsilon(1), \ldots, \widehat{\varepsilon\left(z_{1}\right)}, \ldots, \varepsilon(m)\right)$ and $\beta^{\prime}:\{1, \ldots, m-1\} \rightarrow\{1, \ldots, N\}$ such that $\beta_{k}^{\prime}=\beta_{k}$ if $k=1, \ldots, z_{1}-1$ and $\beta_{k}^{\prime}=\beta_{k+1}$ if $k=z_{1}, \ldots, m-1$.

By hypothesis of induction we know that

$$
\lim _{N \rightarrow \infty} \frac{1}{N^{\frac{m-1}{2}}} \sum_{1 \leq \beta_{1}^{\prime}, \ldots, \beta_{m-1}^{\prime} \leq N}\left\langle A_{\beta_{m-1}^{\prime}}^{\varepsilon^{\prime}(m-1)} \cdots A_{\beta_{1}^{\prime}}^{\varepsilon^{\prime}(1)}\right\rangle=\left\langle A_{0}^{\varepsilon^{\prime}(m-1)} \cdots A_{0}^{\varepsilon^{\prime}(1)}\right\rangle
$$

and the right hand side above is

$$
\left\langle A_{0}^{\varepsilon(m)} \cdots \widehat{A_{0}^{\varepsilon\left(z_{1}\right)}} \cdots A_{0}^{\varepsilon(1)}\right\rangle
$$

As a consequence 5.12 is equal to

$$
\alpha_{d_{\varepsilon}\left(z_{1}\right)}\left\langle A_{0}^{\varepsilon(m)} \cdots \widehat{A_{0}^{\varepsilon\left(z_{1}\right)}} \cdots A_{0}^{\varepsilon(1)}\right\rangle
$$

Corollary 5.1 In the same notations of Theorem 5.1 we have that for any $m \geq 0$

$$
\lim _{N \rightarrow \infty}\left\langle\left(\frac{1}{\sqrt{N}} \sum_{k=1}^{N} Q_{k}\right)^{m}\right\rangle=\int x^{m} d \mu
$$

where, for any $k \in \mathbb{N}$, the operator classical stochastic process $Q_{k}$ is defined by:

$$
Q_{k}=A_{k}+A_{k}^{+}+c_{k} \Lambda_{k}
$$

Proof. For any $m \geq 1$

$$
\left\langle\left(\frac{1}{\sqrt{N}} \sum_{k=1}^{N} Q_{k}\right)^{m}\right\rangle=\frac{1}{N^{\frac{m}{2}}} \sum_{\varepsilon \in\{-1,0,1\}_{+}^{m}} \sum_{1 \leq k_{1}, \ldots, k_{m} \leq N}\left\langle A_{k_{m}}^{\varepsilon(m)} \cdots A_{k_{1}}^{\varepsilon(1)}\right\rangle
$$




$$
\int x^{m} d \mu=\left\langle\left(A_{0}+A_{0}^{+}+\Lambda_{0}\right)^{m}\right\rangle=\sum_{\varepsilon \in\{-1,0,1\}_{+}^{m}}\left\langle A_{0}^{\varepsilon(m)} \cdots A_{0}^{\varepsilon(1)}\right\rangle
$$

Since the sum $\sum_{\varepsilon \in\{-1,0,1\}_{+}^{m}}$ involves only a finite number of terms the thesis follows by applying Theorem 5.1 to each element of the sum.

Acknowledgments The authors want to thank Dr. Anis Ben Ghorbal for useful comments and suggestions. V. C. wants to express his deep gratitude to Dr. Ben Ghorbal also for exciting discussions that greatly improved his understanding of quantum central limit theorems.

\section{References}

[1] Accardi L., Lu Y.G.: Wiener noise versus Wigner noise in quantum electrodynamics, Quantum Probability and Related Topics VIII, (World Scientific, 1993), pp. 1-18.

[2] Accardi L., Lu Y.G., Volovich I.: The QED Hilbert module and interacting Fock spaces, International Institute for Advances Studies, Kyoto (1997).

[3] Accardi L., Bożejko M.: Interacting Fock Spaces and gaussianization of probability measures, Infin. Dimens. Anal. Quantum Probab. Relat. Top. 1, no. 4, pp. 663-670 (1998).

[4] Accardi L., Hashimoto Y., Obata N.: Notions of independence related to the free group, Infin. Dimens. Anal. Quantum Probab. Relat. Top. 1, no. 2, pp. 201-220, (1998).

[5] Accardi L., Ben Ghorbal A., V. Crismale, Y.G. Lu: Projective independence and interacting Fock spaces, in preparation.

[6] Ben Ghorbal A., Schürmann M.: Non-commutative Notions of Stochastic Independence, Math. Proc. Cambridge Philos. Soc. 133, no. 3, pp. 531-561, (2002). 
[7] Ben Ghorbal A., Schürmann M.: Quantum stochastic calculus on Boolean Fock space, Infin. Dimens. Anal. Quantum Probab. Relat. Top. 7, no. 4, pp. 631-650, (2004).

[8] Bercovici H.: A remark on monotonic convolution, Infin. Dimens. Anal. Quantum Probab. Relat. Top. 8 (1) (2005)

[9] Bozejko M., Kümmerer B., Speicher R.: q-Gaussian processes: non commutative and classical aspects, Comm. Math. Phys. 185 (1997) 129154 Preprint (1996)

[10] Bożejko M., Speicher M.: $\psi$-indpendent and symmetrized white noises, Quantum Probability \& related topics, QP-PQ VI, pp. 219-236, (1991).

[11] Cabanal-Duvillard T., Ionescu V.: Un théorème central limite pour des variables aléatoires non-commutatives. Probabilités/Probability Theory, C.R.Acad. Sci. Paris, t. 325, Série 1, pp. 1117-1120 (1997)

[12] Flajolet P.: Combinatorial aspects of continued fractions, Discrete Math. 32, no. 2, pp. 125-161, (1980).

[13] Franz U.: Unification of Boolean, monotone, anti-monotone, and tensor independence and Lévy processes, Math. Z. 243, no. 4, pp. 779-816, (2003).

[14] Hashimoto Y.: A construction of interacting Fock spaces derived from quantum decomposition and its applications, Infinite dimensional analysis and quantum probability theory (Japanese) (Kyoto, 2000). Sūrikaisekikenkyūsho Kōkyūroku No. 1227 (2001) 124-139

[15] Hashimoto Y.: Quantum decomposition in discrete groups and interacting Fock spaces, Infin. Dimens. Anal. Quantum Probab. Relat. Top. 4, pp. 277-287, (2001).

[16] Hashimoto Y., Hora A., Obata N.: Central limit theorems for large graphs: Method of quantum decomposition, J. Math. Phys. 44, pp. 7188, (2003).

[17] Lenczewski R.: Reduction of free independence to tensor independence, Infin. Dimens. Anal. Quantum Probab. Relat. Top. 7, no. 3, pp. 337-360, (2004). 
[18] Lu Y.G.: On the interacting free Fock space and the deformed Wigner law, J. Nagoya Math. 145, pp. 1-28, (1997).

[19] Lu Y.G.: Interacting Fock spaces related to Anderson model, Infin. Dimens. Anal. Quantum Probab. Relat. Top. 1, no. 2, pp. 247-287, (1998).

[20] Mlotkowski W.: Free probability on algebras with infinitely many states, Probab. Theory Related Fields 115, no. 4, pp. 579-596, (1999).

[21] Muraki N.: Noncommutative Brownian motion in monotone Fock space, Comm. Math. Phys. 183, no. 3, pp. 557-570, (1997).

[22] Muraki N.: The Five independences as natural products, Infin. Dimens. Anal. Quantum Probab. Relat. Top. 6 , no. 3, 337-371, (2003).

[23] Schürmann M.: Non-commutative probability on algebraic structures, Probablity measures on groups and related structures XI, (World Scientific, 1995), pp. 332-356.

[24] Speicher, R.: A new example of "independence" and "white noise", Probab. Theory Relat. Fields 84, no. 2, pp. 141-159, 1990.

[25] Speicher R., Woroudi R.: Boolean convolution, Free Probability theory, Fields Inst. Commun. 12, pp. 267-279, (1997).

[26] Speicher R. : On universal product, Free Probability theory, Fields Inst. Commun., 12, pp. 257-266, (1997).

[27] Voiculescu D., Dykema K., Nica A.: Free Random Variables, CRM Monographs Series, 1, AMS, (1992). 